ORIGINAL ARTICLE

\title{
Clinical features and follow up of 302 patients with Mycobacterium kansasii pulmonary infection: a 50 year experience
}

\author{
N Maliwan, J R Zvetina
}

Postgrad Med J 2005;81:530-533. doi: 10.1136/pgmi.2004.026229

See end of article for authors' affiliations .....................

Correspondence to: Dr N Maliwan, PO Box 1017, VAH, Hines, Illinois 60141,USA; pitsanulok@ aol.com

Submitted 29 June 2004 Accepted 27 October 2004

\begin{abstract}
Aims: To analyse clinical features and treatment outcomes of patients with pulmonary Mycobacterium kansasii infection treated at Hines VA Hospital between 1952 and 1995, and followed up until 2003. Findings: 302 patients were confirmed to have $M$ kansasii pulmonary infection; diagnosis was not made until death in $2 \%$. The average age was 50 years old; $76 \%$ were white; all were men. Productive cough, dyspnoea, and chest pain were common; $16 \%$ were asymptomatic. Right sided, apical or subapical, thin walled cavitary infiltrate was the characteristic radiological feature. Heavy smoking, chronic obstructive pulmonary disease, alcoholism, peptic ulcer disease, coronary artery disease, prior tuberculosis, psychosis, prior pneumonia, and immunocompromising conditions were prevalent. Average follow up was 10 years and 2 months. PPD was positive in $58 \%$ of 179 tested. Two thirds of the patients required only first line drugs. Fourteen per cent required surgical intervention, none after 1977. Spontaneous resolution occurred in 1\%. Aspergillosis developed in 4\%. Bronchogenic carcinoma coexisted with $M$ kansasii infection in $6 \%$ and followed it in $4 \%$. Extrapulmonary malignancy coexisted with the infection in $4 \%$ and followed it in 6\%; most involved head and neck. Eleven per cent of 224 deaths were attributed to $M$ kansasii. Outcomes were affected by comorbidity, treatment compliance, rifampicin use, and extent of infection.

Conclusions: Prognosis of $M$ kansasii pulmonary infection is good if diagnosed and treated early, together with control of underlying conditions. Clinicians should be aware of atypical radiological manifestations of the disease when coexisting with other pulmonary or immunocompromising conditions.
\end{abstract}

$T$ he role of Mycobacterium kansasii as an infecting organism was not recognised until the early 1950s. M kansasii infection shares many clinical aspects with tuberculosis (TB). However, $M$ kansasii infection contrasts with TB by being non-contagious, more associated with underlying diseases, and having a definite geographical distribution-that is, in southern and central USA. ${ }^{1-4}$ In a non-endemic area, a higher incidence of $M$ kansasii isolates was found among HIV positive persons, and more predisposing medical conditions were found among HIV negative persons. ${ }^{5}$ While the prevalence of TB has decreased in the past 20 years, those of non-tuberculous mycobacterial infections including $M$ kansasii have been increasing worldwide. As a positive sputum culture for $M$ kansasii may only reflect colonisation, strict criteria have been developed for the diagnosis of pulmonary $M$ kansasii infection to exclude a mere colonisation. ${ }^{67}$

Varying findings on $M$ kansasii pulmonary infection were reported from cancer treatment centres, HIV treatment centres, TB centres, mining company TB clinics, and pulmonary departments. We present here data analysis of the largest patient population with the longest follow up, hopefully to confirm prior findings and to help resolve some of the conflicting conclusions.

\section{METHODS}

This is a retrospective study of $M$ kansasii pulmonary infection cases treated at the Veterans Administration Hospital, Hines, Illinois. This study was originated as part of "the 1962 Protocol 6: disease due to mycobacteria other than M. TBC" by the Veterans Administration and Armed Forces, with the cooperation of the National Tuberculosis Association.

We reviewed the medical records and radiographs of all patients with positive sputum cultures for $M$ kansasii between
1952 and 1995. The recommended criteria by the American Thoracic Society were used in making the diagnosis of $M$ kansasii pulmonary infection, for the inclusion in this study. For patients with cavitary lung disease, at least two positive cultures of moderate to heavy growth must be present, while other reasonable causes for the disease process have been excluded; for patients with non-cavitary lung disease, those cultures must fail to clear with bronchial toilet or two weeks of antimycobacterial therapy; and, patients whose sputum evaluation is non-diagnostic or another disease cannot be excluded must have: the organism and granulomatous inflammation from a lung biopsy, or two or more positive sputum cultures plus granulomatous features without other reasonable causes. ${ }^{6}$

Data collected were: demographic, date of diagnosis, presenting symptoms, radiological findings, reaction to intermediate strength (5 TU) PPD skin test, underlying conditions, treatment modalities and outcomes, last follow up date, and cause of death on those who have died. The presence of malignancy, its histology, location, and temporal relation to $M$ kansasii were determined.

\section{RESULTS}

A total of 302 patients admitted between 1952 and 1995 were confirmed to have active $M$ kansasii pulmonary infection, with an annual average of seven (range 0-37). All were men, 231 $(76 \%)$ were white, 69 (23\%) were black, and one each was Latin American and Asian American. The average age was 50 years (range 23-84).

\section{Clinical presentation}

The main symptoms were cough $(84 \%)$, sputum production $(80 \%)$, shortness of breath $(32 \%)$, and chest pain $(28 \%) ; 16 \%$ 
were asymptomatic. Haemoptysis was present in 72 patients (24\%), mostly in the form of blood streaked sputum. The syndrome of inappropriate secretion of antidiuretic hormone was present in two patients. Diagnosis was prompted by radiological changes during a routine follow up of 16 patients who had a pulmonary or extrapulmonary malignancy. The disease was discovered through a routine admitting chest radiograph in 32 other patients: seven for acute coronary syndrome, seven for chronic obstructive pulmonary disease (COPD), five for gastrointestinal bleeding, three for alcoholism, three for coexisting pulmonary malignancy, three for elective surgery (one each for hip replacement, inguinal hernia repair, and fractured bone), two for neuropathy, and one each for bone malignancy and schizophrenia.

Positive PPD reaction of $10 \mathrm{~mm}$ or more to a 5-TU PPD was found in 105 of the 179 patients tested (59\%). Among the negative reactors, three patients were given additional testing; two had a positive test to a 250-TU PPD, and one had a positive reaction to a PPD from $M$ kansasii.

\section{Radiological findings}

The presence of underlying pulmonary conditions-that is, emphysematous bullae, old TB scarring, malignancy, radiation pneumonitis, pneumoconiosis, and prior lung surgery, masked the radiological findings of $M$ kansasii infection initially in many patients.

All 302 patients had an abnormal chest radiograph at the time of diagnosis. Only three patients had lesions that spared the upper lobes: one patient with middle and lower lobe involvement had a history of pleural effusion and pneumothorax, one had a history of upper lobectomy for TB, and one had a contralateral upper lobe $M$ kansasii lesion.

The extent of the disease was minimal (total area of lesions less than one third of one lung with no cavitation) in 22 patients (7\%); moderately advanced (total area from one third of a lung to one lung, total diameter of cavity, if present, less than $4 \mathrm{~cm}$ ) in 159 (53\%); and far advanced (more

Table 1 Underlying conditions: 302 total M kansasii patients compared with $24 \mathrm{M}$ kansasii deaths

\begin{tabular}{|c|c|c|}
\hline Conditions & $\begin{array}{l}\text { Total number } \\
(\%)\end{array}$ & $\begin{array}{l}\text { M kansasii } \\
\text { deaths number } \\
(\%)\end{array}$ \\
\hline Heavy smoking & $220(73)$ & $20(83)$ \\
\hline COPD & $197(65)$ & $23(96)$ \\
\hline Alcohol misuse & $170(56)$ & $14(58)$ \\
\hline Peptic ulcer disease & $60(20)$ & $5(21)$ \\
\hline Coronary artery disease & $60(20)$ & 7 (29) \\
\hline Prior TB & 43 (14) & $7(29)$ \\
\hline Psychosis & 38 (13) & $5(21)$ \\
\hline Prior pneumonia & $36(12)$ & $10(42)$ \\
\hline Cirrhosis & $26(9)$ & $1(4)$ \\
\hline Diabetes mellitus & $20(7)$ & $2(8)$ \\
\hline Bronchogenic carcinoma & $17(6)$ & $2(8)$ \\
\hline Congestive heart failure & $16(5)$ & $3(13)$ \\
\hline Extrapulmonary malignancy & $13(4)$ & $2(8)$ \\
\hline Cor pulmonale & $9(3)$ & $4(17)$ \\
\hline Rheumatoid arthritis & $8(3)$ & $1(4)$ \\
\hline Cholecystectomy & $8(3)$ & $1(4)$ \\
\hline Malnutrition & $8(3)$ & $6(25)$ \\
\hline AIDS & $7(2)$ & - \\
\hline Bronchiectasis & $7(2)$ & $1(4)$ \\
\hline Asthma & $6(2)$ & $2(8)$ \\
\hline $\begin{array}{l}\text { Intravenous drug misuse (four with } \\
\text { AIDS) }\end{array}$ & $6(2)$ & - \\
\hline Silicosis & $5(2)$ & $1(4)$ \\
\hline Corticosteroid use & $3(1)$ & $1(4)$ \\
\hline Organ transplant* & $3(1)$ & - \\
\hline Asbestosis & $2(1)$ & $1(4)$ \\
\hline Sarcoidosis & $2(1)$ & 1 (4) \\
\hline Healed histoplasmosis & $2(1)$ & - \\
\hline
\end{tabular}

Table 2 Malignancy coexisting with or after M kansasii infection

\begin{tabular}{|c|c|c|}
\hline & $\begin{array}{l}\text { CA coexists } \\
\text { with } M \\
\text { kansasii }\end{array}$ & $\begin{array}{l}\text { CA follows } \\
M \text { kansasii }\end{array}$ \\
\hline \multicolumn{3}{|l|}{ Bronchogenic CA } \\
\hline Squamous cell & 8 & 6 \\
\hline Adeno & 2 & 3 \\
\hline Undifferentiated or poorly differentiated & 2 & 1 \\
\hline Miscellaneous & $1^{*}$ & $2 \dagger$ \\
\hline Subtotal & 13 & 12 \\
\hline \multicolumn{3}{|l|}{ Extrapulmonary malignancy } \\
\hline Gastrointestinal & 6 & 2 \\
\hline Head and neck & 4 & 9 \\
\hline Genitourinary & 4 & 7 \\
\hline Miscellaneous & $1 \neq$ & $3 \S$ \\
\hline Subtotal & 15 & 21 \\
\hline \multicolumn{3}{|c|}{$\begin{array}{l}\text { *Small cell CA. †One each of mixed squamous and adenoCA and } \\
\text { bronchogenic CA without specification. } ¥ \text { Acute leukaemia. } § \text { One each o } \\
\text { small cell lymphoma; small cell undifferentiated CA of mediastinal lympl } \\
\text { node without primary; and adenoCA of lungs, lymph nodes and liver } \\
\text { without a primary. }\end{array}$} \\
\hline
\end{tabular}

extensive than moderately advanced) in the remaining 121 patients $(40 \%)$.

In 128 patients (42\%) the infiltrates were bilateral: 58 had bilateral cavities, 38 had only right lung cavitation, 21 had only left lung cavitation, and 11 with no cavity. Unilateral right lung infiltrates were the presenting findings in 105 patients (35\%), 87 of which had cavitation. The remaining 69 patients $(23 \%)$ had unilateral left lung infiltrates, 58 of which contained cavitation. Single cavitary lesions accounted for 140 patients $(46 \%)$, multiple cavitary lesions for 122 patients $(40 \%)$, and no cavity for 40 patients (13\%). All seven AIDS patients with confirmed $M$ kansasii pulmonary infection had infiltrates without any cavitation.

\section{Underlying conditions}

Heavy cigarette smoking, COPD, and alcohol misuse each was present in more than half of the patients in our series (table 1).

\section{Underlying malignancies}

Bronchogenic carcinoma (CA) was reported in 17 patients. In four patients, the CA (two adenoCA, one small cell CA, and

Table 3 Cause of deaths: 223 cases of pulmonary $M$ kansasii infection

\begin{tabular}{ll}
\hline Cause of death & Number (\%) of patients \\
\hline Coronary artery disease & $40(18)$ \\
Bacterial pneumonia & $29(13)$ \\
M kansasii & $24(11)$ \\
COPD & $23(10)$ \\
CA lung & $18(8)$ \\
Other malignancies & $18(8)$ \\
Congestive heart failure & $11(5)$ \\
Alcoholic liver disease & $10(4)$ \\
AlDS & $6(3)$ \\
Pulmonary embolism & $6(3)$ \\
Sepsis & $5(2)$ \\
Bleeding peptic ulcer disease & $4(2)$ \\
Trauma & $4(2)$ \\
Cerebrovascular accident & $3(1)$ \\
Disseminated aspergillosis & $2(1)$ \\
Pancreatitis & $2(1)$ \\
Miscellanous* & $7(3)$ \\
Unknown & $11(5)$ \\
\hline *One case each for bleeding secondary to autoimmune \\
thrombocytopenia, hepatitis C, candidiasis, subacute bacterial \\
endocarditis, sarcoidosis, haemoptysis, and drug reaction.
\end{tabular}


one malignant fibrohistiocytoma) had been present months or years before $M$ kansasii; three of these patients had received radiation therapy, with or without chemotherapy and corticosteroids. ${ }^{8}$ In 13 patients, bronchogenic CA was found to coexist with active $M$ kansasii infection (table 2); the average age was 56 years; nine were white and four were black. M kansasii and CA lesions were located in the same lung in eight patients. Radiological appearances of these coexisting pulmonary malignancies were: a mass, atelectasis, nodular lesion, or infiltrates. Squamous cell CA was the most common histological finding $(62 \%)$. One patient was also found to have another primary tumour (laryngeal CA) while still active with $M$ kansasii.

$M$ kansasii pulmonary infection was diagnosed along with extrapulmonary malignancy, excluding skin cancers, in 13 patients; two of them also had a second primary tumour while $M$ kansasii was still active. The most common site of these extrapulmonary coexisting CA was gastrointestinal, followed by head and neck. The most common cell type was squamous cell $(40 \%)$. Active $M$ kansasii infection occurred during radiation therapy alone in seven patients, and during both radiation and chemotherapy in one patient.

\section{Treatment}

(1) No treatment: 10 patients had never received any antimycobacterial treatment. $M$ kansasii pulmonary infection was not diagnosed until death in five patients; none died because of $M$ kansasii. Two patients refused treatment. The culture reports for the remaining three patients were not available until after their discharges. Gradual resolution of the infiltrates on chest radiographs along with conversion to negative cultures was reported in four patients, including one of the two patients who had refused antimycobacterial therapy. Another patient who had refused the treatment later died from AIDS.

(2) Medical treatment: among the 292 patients who were treated, $201(69 \%)$ received only two or three first line antimycobacterial drugs (ethambuthol, isoniazid, paraaminosalicylic acid, pyrazinamide, rifampicin, and streptomycin). The remaining 91 patients also required some of the second line drugs (amikacin, capreomycin, cycloserine, ethionamide, kanamycine, and viomycin).

The duration of the antimycobacterial treatment was 1824 months for most cases. Longer treatment was needed for those with relapses, mainly as a result of poor compliance or the presence of malignancy.

(3) Surgical treatment: combined medical-surgical treatment was necessary in 42 patients (14\%) for a total of 53 surgical procedures. All but five were performed before 1970, and none were after 1977. A preliminary report was made on 35 of these patients. ${ }^{9}$ Lobectomy was performed in 30 patients, lung resection in eight patients, pneumonectomy and wax-pack plombage in five patients each, thoracoplasty in four patients, and one patient had decortication. The indications for surgery were: residual cavity, medical failure, or both. $M$ kansasii culture was positive at the time of surgery for 31 procedures (58\%). Postoperative complications included atelectasis in three patients, and one patient each for empyema, bleeding, and pneumothorax. Seven patients had postoperative relapses; all subsequently achieved inactive status on follow up.

\section{Outcomes}

Two hundred and twenty three patients (74\%) were followed up until death. Eight patients were alive and free of $M$ kansasii as of May 2003. The remaining 71 patients either moved away to other states or were otherwise lost from follow up; all but two had sputum conversion by the last follow up. Excluding the five patients whose diagnosis of $M$ kansasii was not made until death, the average length of follow up was 10 years, 2 months, and 11 days (range 1 day to 40 years, 7 months, and 27 days).

Most patients cleared the organism within four to six months of medical treatment. Before the use of rifampicin, the six month sputum conversion rate was $73 \%$; only $19 \%$ of sputum cultures remained positive after three months of rifampicin based drug combination. Two patients from the prerifampicin group never converted on multiple drug combinations, and died three and eight years later from disseminated $M$ kansasii infection and $M$ kansasii empyema, respectively.

Bronchogenic CA developed in 12 patients with inactive $M$ kansasii. Its location was the same as that of $M$ kansasii in seven patients. Squamous cell CA was the most common histological finding (50\%). The CA followed $M$ kansasii diagnosis by 4 to 26 years. Three of these cases remain alive. For the nine cases who had died, the cause of death was CA; none had active $M$ kansasii at the time of death.

Twenty one extrapulmonary malignant tumours developed after the completion of $M$ kansasii treatment in 20 patients. The CA were diagnosed two to nine years after the treatment of $M$ kansasii. Squamous cell CA of the head and neck was the most common CA in this group (43\%), followed by adenoCA of the prostate. One patient had both CA larynx and CA rectum before death.

Aspergillous infection was found in 13 patients. In 12 patients, it followed the treatment of $M$ kansasii pulmonary infection by many years. The remaining patient, whose aspergilloma and $M$ kansasii pulmonary infection were diagnosed at the same time, had pre-existing TB scars. Ten patients developed mycetoma, seven of which were reported earlier. ${ }^{10}$ All but one aspergilloma originated within a large, inactive $M$ kansasii cavity; all but two were located in the right upper lobe. Two patients developed disseminated aspergillosis while receiving immunosuppressive therapy for a heart transplant: One also had pulmonary candidiasis with candidaemia, and the other had chronic aspergillous pneumonia.

Pulmonary TB was found to follow inactive $M$ kansasii in only one patient in our series. ${ }^{11}$

\section{Mortality}

Among the 223 patients who were followed up until death, a complete necropsy report was only available on 64 . Table 3 shows the more frequent causes of death for the 223 patients to be: coronary artery disease, bacterial pneumonia, $M$ kansasii, COPD, malignancies, and congestive heart failure.

Although $M$ kansasii was active at the time of death in 56 patients it was the cause of death in only 24, two of whom had $M$ kansasii empyema ${ }^{12}$ and one had disseminated $M$ kansasii infection to the lungs, kidneys, and peritoneum. The causes of death in 32 other patients with active $M$ kansasii were: COPD (seven patients), coronary artery disease (five patients), bronchopneumonia (five patients), pulmonary emboli (three patients), bleeding peptic ulcer (three patients), CA colon (three patients), and one each for CA stomach, combination of CA tonsil and CA rectum, lung abscess, alcoholic cirrhosis with oesophageal bleeding, AIDS, and disseminated aspergillosis.

Among the 24 patients who died from $M$ kansasii, 17 had far advanced disease and seven had moderately advanced disease at the time of the diagnosis. Only five patients were diagnosed after 1970.

\section{DISCUSSION}

Our largest scale study with longest term follow up of $M$ kansasii pulmonary infection helps shed more light on the natural history of this infection. It confirmed the preponderance among white, middle aged men, heavy smokers, and a 
high association with COPD, alcohol misuse, prior TB, gastrectomy, malignancies, and other immunocompromising conditions. The presence of all men in our $M$ kansasii patients also reflects the $98 \%$ male preponderance at our Veterans Hospital. The high prevalence of PPD positivity was not likely to have resulted from prior TB or BCG exposure, as only five of the 105 positive reactors had a history of TB and none had a history of BCG vaccination. We believe cross activity with $M$ kansasii was the probable explanation.

There were conflicting reports regarding the radiological similarities and dissimilarities between $M$ kansasii and TB in terms of location, number, size, and wall thickness of the cavities, etc. ${ }^{17^{13} 14}$ We found an $M$ kansasii pulmonary lesion to have a striking radiological pattern. A thin walled cavity, surrounded by a small amount of parenchymal infiltrate, located in an apical or subapical area, mainly of the right lung, with a pleural reaction, is very likely to be an $M$ kansasii rather than a TB lesion. Samples of these typical radiological appearances from our experience have been published earlier. ${ }^{15}$ Underlying lung conditions as well as other immunocompromising diseases can, however, significantly distort the radiological presentation.

The associations between $M$ kansasii pulmonary infection and malignancy were reported mainly from cancer treatment centres. The $M$ kansasii infection usually followed malignancy, with chemotherapy or radiation therapy as additional predisposing conditions. ${ }^{16}{ }^{17}$ Our series included four cases of bronchogenic CA that clearly preceded $M$ kansasii, and 13 additional cases that coexisted with $M$ kansasii, with a total association rate of $6 \%$. This association posed serious diagnostic problems. Both conditions often involve middle aged men with COPD and heavy cigarette smoking. Many of their radiological features also overlap; advanced $M$ kansasii can manifest as diffuse infiltrates with or without any cavity, ${ }^{6}$ while cavitation in lung cancers occurs in $2 \%-16 \%$ of cases and an infiltrative pattern is also common among a certain CA. ${ }^{18}$ Moreover, radiation treatment of the CA could result in radiation pneumonitis or fibrosis. Indeed, the coexistence of $M$ kansasii and CA in our series was recognised during the initial evaluation in only three patients; in three other patients the tumours were unmasked only after months of antimycobacterial therapy. Diagnostic delay may have contributed to death in some of these patients.

The high incidence of pulmonary and extrapulmonary CA, especially of the head and neck, in our series might simply reflect the high prevalence of heavy smokers in our series. The high prevalence of bronchogenic CA locating at the old $M$ kansasii site, however, suggests the possibility of scar tumours, similar to those after TB.

Similar to other reports, ${ }^{16719}$ most of our patients responded well to antimycobacterial regimens, and rarely required surgical treatment, especially since the inclusion of rifampicin. The use of rifampicin in the treatment of $M$ kansasii infection at our institution started in 1966, but did not become part of the routine drug combination until 1971.

Even though this is a retrospective study, the incidences of underlying conditions were noted to be quite high compared with those from most other reports. As shown in table 1, we also found certain conditions to be significant comorbidities, namely, COPD, cor pulmonale, prior pneumonia, prior TB, psychosis, and malnutrition. Whether the high incidence of prior pneumonia reflects a pre-existing impaired defence mechanism in the patient's lungs is unknown. The death rate of $M$ kansasii pulmonary infection at $11 \%$ in our series was rather high. This could be attributable to several factors: (1) our study period also covered several years before the use of rifampicin; (2) the high prevalence of advanced disease at the time of diagnosis; and (3) the high incidence of alcoholism and psychiatric disorders, leading to poor treatment compliance.

\section{CONCLUSION}

For the best possible therapeutic outcome, $M$ kansasii should be actively investigated in certain high risk patients. Middle aged, white men with a heavy smoking history and COPD, from an $M$ kansasii endemic area, who present with a thin walled cavitary lesion in an apical or subapical area, should be investigated for $M$ kansasii. Appropriate treatment combinations should include rifampicin. As $M$ kansasii may coexist with pulmonary malignancy, a diagnosis of malignancy should not automatically exclude the diagnosis of $M$ kansasii, but rather alert the clinicians to the possibility of atypical radiological presentation. With an early diagnosis, $M$ kansasii pulmonary infection can usually be treated effectively.

\section{ACKNOWLEDGEMENTS}

We thank Joseph Lentino, MD, PhD and Constance Pachucki, MD, for recent case referrals; Lawrence Brand for the initial data collection; and Ralph W Doran for manuscript preparation.

\section{Authors' affiliations}

N Maliwan, Loyola University Medical Center, Maywood, Illinois, USA J R Zvetina, Abraham Lincoln School of Medicine, University of Illinois, USA

Funding: none.

Conflicts of interest: none declared.

Institution where the work was performed: the Veterans Administration Hospital, Hines, Illinois, USA.

\section{REFERENCES}

1 Lillo M, Orengo S, Cernoch P, et al. Pulmonary and disseminated infection due to Mycobacterium kansasii: a decade of experience. Rev Infect Dis 1990;12:760-7.

2 O'Brien RJ. The epidemiology of nontuberculous mycobacterial disease. Clin Chest Med 1989; 10:407-18.

3 Good RC, Snider DE Jr. Isolation of nontuberculous mycobacteria in the United States, 1980. J Infect Dis 1982;146:829-33.

4 Bittner MJ, Horowitz EA, Safranek TJ, et al. Emergence of Mycobacterium kansasii as the leading mycobacterial pathogen isolated over a 20-year period at a midwestern Veterans Affairs Hospital. Clin Infect Dis 1996;22:1109-10.

5 Bloch KC, Zwerling L, Pletcher MJ, et al. Incidence and clinical implications of isolation of Mycobacterium kansasii: result of a 5-year, population-based study. Ann Intern Med 1998;129:698-704.

6 Wallace RJ Jr, O'Brien R, Glassroth J, et al. Diagnosis and treatment of disease caused by nontuberculous mycobacteria. Am Rev Respir Dis 1990;142:940-53.

7 Wallace RJ Jr, Glassroth J, Griffith DE, et al. Diagnosis and treatment of disease caused by nontuberculous mycobacteria. Am J Respir Crit Care Med 1997; 156:S1-25.

8 Zvetina JR, Maliwan N, Frederick WE, et al. Mycobacterium kansasii infection following primary pulmonary malignancy. Chest 1992;102:1460-3.

9 Zvetina JR, Neville WE, Maben HC, et al. Surgical treatment of pulmonary disease due to Mycobacterium kansasii. Ann Thorac Surg 1971;1 1:551-6.

10 Maliwan N, Zvetina JR. Pulmonary mycetoma following Mycobacterium kansasii infection: report of seven cases. Arch Intern Med 1985;145:2180-3.

11 Zvetina JR, Nathan N, Facen H. Pulmonary tuberculosis following successful treatment of pulmonary infection with Mycobacterium kansasii. Chest 1976;70:786-8.

12 Zvetina JR, Difilippo NM, Ali MM, et al. Mycobacterium kansasii empyema. Tubercle $1981 ; 62: 135-8$.

13 Spitz HB, Wiot JF. Nontuberculous mycobacterial pulmonary disease. Semin Roentgenol 1979;14:244-8.

14 Papillon F, Huchon G, Labrune S, et al. Non-tuberculous mycobacterial disease of the lung in a pulmonology department. Bull Int Union Against Tuber Lung Dis 1988;63:17-19.

15 Zvetina JR, Demos TC, Maliwan N, et al. Pulmonary cavitations in Mycobacterium kansasii: distinctions from M. tuberculosis. AJR Am J Roentgenerol 1984; 143:127-30.

16 Feld R, Bodey GP, Groschel D. Mycobacteriosis in patients with malignant disease. Arch Intern Med 1976;136:67-70.

17 Jacobson KL, Teira R, Libshitz HI, et al. Mycobacterium kansasii infections in patients with cancer. [Corrected and republished from Clin Infect Dis 2000;30:965-9]. Clin Infect Dis 2000;31:628-31.

18 Woodring JH. Piffalls in the radiologic diagnosis of lung cancer. AJR Am J Roentgenerol 1990;154:1165-7.

19 Campbell IA, Drobniewski F, Novelli V, et al. Management of opportunist mycobacterial infections: Joint Tuberculosis Committee guidelines 1999 Thorax 2000;55:210-18. 